\title{
BMJ Open Study on acupuncture in the treatment of painful diabetic peripheral neuropathy based on rs-fMRI: a protocol for systematic review and meta-analysis
}

\author{
Mengyuan Li (1) , Lin Yao (D), Haipeng Huang, Haizhu Zheng, Jiazhen Cao, \\ Guan Wang, Yanze Liu, Hongfeng Wang
}

To cite: Li M, Yao L, Huang H, et al. Study on acupuncture in the treatment of painful diabetic peripheral neuropathy based on rs-fMRl: a protocol for systematic review and meta-analysis. BMJ Open 2021;11:e055874. doi:10.1136/ bmjopen-2021-055874

- Prepublication history for this paper is available online. To view these files, please visit the journal online (http://dx.doi org/10.1136/bmjopen-2021 055874).

Received 26 July 2021 Accepted 09 August 2021

Check for updates

(C) Author(s) (or their employer(s)) 2021. Re-use permitted under CC BY-NC. No commercial re-use. See rights and permissions. Published by BMJ.

College of Acupuncture and Massage, Changchun University of Chinese Medicine, Changchun, Jilin, China

Correspondence to Professor Hongfeng Wang; ccwhf@126.com

\section{ABSTRACT}

Introduction Studies have shown that acupuncture has significant therapeutic effects on painful diabetic peripheral neuropathy (PDPN) yet the precise mechanism of action underpinning these effects remains controversial. Resting-state functional MRI (rs-fMRI) is an advanced imaging technique that can be used to monitor changes in the activity of the brain, particularly in PDPN. However, the data from several studies remain inconclusive and there is currently no systematic review and meta-analysis for the use of rs-fMRI in PDPN.

Methods and analysis In this study, we will select all eligible studies published on or before 30 June 2021. Four English and four Chinese databases will be searched, specifically, PubMed, Embase, Web of Science, Cochrane Library, China National Knowledge Infrastructure, WanFang database, China Science Technology Journal Database (VIP) and China Doctor/Master Dissertations Full-text Database. Only clinical trials and the first cycle of a crossover trial linked to acupuncture for PDPN will be included in the analysis. The main outcomes include the amplitude of low-frequency fluctuation, regional homogeneity, functional connectivity of the brain, bilateral superficial peroneal nerve sensory nerve conduction velocity, bilateral dorsal current perception threshold values and the degree of subjective pain. The secondary outcomes include biochemical indicators, the degree of depression and anxiety and changes in efficiency. The study selection, data extraction and risk of bias assessment will be performed by two investigators. For statistical analyses, Review Manager V.5.4 software will be used. If necessary, heterogeneity testing, data synthesis, and subgroup analysis will be performed.

Ethics and dissemination Our systematic review and meta-analysis will be based on published literature for data extraction and will not include the use of individual patient data and so no ethical approval required.

PROSPERO registration number CRD42021211644.

\section{INTRODUCTION}

Diabetic peripheral neuropathy (DPN) refers to the presence of signs and/or symptoms associated with peripheral nerve dysfunction in patients with diabetes when other causes are excluded. ${ }^{1}$ It is one of the most common
Strengths and limitations of this study

- This study will be the first systematic review and meta-analysis to investigate the effects of acupuncture based on brain activity of painful diabetic peripheral neuropathy.

- Strict inclusion and exclusion criteria will be used to check for randomised controlled trials in four English databases and four Chinese databases.

- To verify the robustness of the conclusions, sensitivity analysis will be performed.

- Any missing or inadequate data will be acquired by contacting the first or corresponding author of the included studies.

- Heterogeneity may occur across different acupuncture methods leading to difficulties in data collation.

complications of diabetes mellitus (DM) and occurs in $46.6 \%$ of patients with diabetes in China. ${ }^{2}$ Painful diabetic peripheral neuropathy (PDPN) is a disease for which there are currently no effective treatments and has a major impact on the quality of life of patients with diabetes. ${ }^{3}{ }^{4}$ The prevalence of PDPN increases with age. ${ }^{5}$ Around $12 \%$ of patients with PDPN do not report significant symptoms and $39 \%$ of patients do not receive any treatment. ${ }^{6}$ The main clinical manifestations of PDPN are numbness, tingling and abnormal sensations mostly in the lower extremities and increased pain at night. More than twothirds of patients with PDPN suffer from varying degrees of depression, anxiety and sleep disorders ${ }^{78}$ resulting in a major societal burden on families and caregivers. The latest research data have shown that diabetes and its complications are also important risk factors for the progression and adverse outcomes of patients with COVID-19. ${ }^{9}$

The pathogenesis of PDPN is complex involving metabolic disorders, vascular damage, neurological damage, oxidative 
stress damage and the inflammatory response. Although poor control of blood glucose is the most important cause of PDPN, its exact aetiology remains unclear. Several studies have indicated that peripheral and central sensitisation play an important role in the onset and progression of PDPN. ${ }^{10-12}$ Peripheral sensitisation involves the hypersensitisation of primary injury receptor neurons resulting in an enhanced nociceptive response to normal pain-causing stimuli. In contrast, central sensitisation refers to the amplification of the response of the spinal cord and the central nervous system above the spinal cord to pain transmission. Central sensitisation is evidenced by the expansion of the receptive field, enhanced response to stimuli, prolongation of the duration and lowering of the pain threshold. It has been shown that dysfunction of the descending pain modulation system mediated by the ventrolateral periaqueductal grey-mediated may reflect a brain-based pain promotion mechanism leading to PDPN. ${ }^{13}$ Therefore, this deleterious injury should be addressed early to prevent the continued exacerbation of neurological complications.

The pathogenesis of PDPN remains unclear and so it is difficult for modern medicine to develop improved treatment options for PDPN. Approaches such as control of blood glucose, nerve nutrition and pain treatment can be used but often deliver unsatisfactory outcomes. ${ }^{3}$ Pain associated with PDPN is often treated with tricyclic antidepressants (amitriptyline, promethazine and nortriptyline), 5-hydroxytryptamine and norepinephrine reuptake inhibitors (duloxetine, griseofulvin), anticonvulsants (gabapentin, pregabalin) and opioids. However, only duloxetine and pregabalin are currently approved by the US Food and Drug Administration and the European Medicines Agency for the treatment of PDPN. ${ }^{14}$ Although a variety of drugs are available for the treatment of PDPN, none has been shown to provide $100 \%$ effective relief from symptoms and are also associated with significant adverse side effects. ${ }^{1516}$

Acupuncture is an important component of traditional Chinese medicine that has demonstrated significant advantages in the treatment of PDPN. ${ }^{17}$ Acupuncture involves the transmission of information through body surface stimulation to the brain center for integration and processing which in turn outputs instructions that regulate the neuroendocrine-immune system and overall body function. ${ }^{18}$ Acupuncture has been shown to improve the state of the body and can be used to treat many diseases. Studies have shown that acupuncture can significantly improve pain in patients with PDPN by reducing their symptoms of burning and tingling sensations, numbness and heterogeneous pain. ${ }^{19}{ }^{20}$ Acupuncture is a complex intervention and while some small-sample randomised controlled clinical trials have demonstrated efficacy in the treatment of $\mathrm{PDPN}_{2}$ the specific mechanisms remain to be fully understood. Resting-state functional MRI (rs-fMRI) has been widely used to study neurological disorders as it assesses the activity of the central nervous system. The amplitude of low-frequency fluctuation (ALFF) is a validated rs-fMRI analysis method that can be used to assess the neuronal activity of the brain by measuring the low-frequency oscillation $(0.01-0.08 \mathrm{~Hz})$ in the blood oxygen level dependent (BOLD) signal. ${ }^{21}{ }^{22}$ Regional homogeneity (ReHo) is a method that allows local correlations in the BOLD signal to reveal regional synchronisations of temporal changes in BOLD. ${ }^{23}$ Seedbased functional connectivity (FC) analysis is also used to investigate abnormal FC between areas of the brain in the PDPN. Studies have evaluated abnormal brain activity in patients with diabetic neuropathy treated with acupuncture using ALFF, ReHo and FC yet these studies have reported inconclusive findings. ${ }^{24-28}$ Moreover, previous studies on acupuncture for PDPN have been focused on changes in the spinal level rather than the central level due to the limitations of research techniques. Recently, rapid developments in rs-fMRI technology have allowed it to be widely used to investigate changes in brain function changes in a variety of diseases. We hypothesise that this technique could be successfully applied to investigate changes in the brain in the treatment of PDPN with acupuncture.

\section{METHODS}

\section{Study registration}

This study is registered on the International Platform of Registered Systematic Review and Meta-analysis Protocols (PROSPERO) and is reported in compliance with the Preferred Reporting Items for Systematic Reviews and Meta-Analysis (PRISMA) Protocol statement guidelines. ${ }^{29}$

\section{Patient and public involvement}

No patient involved.

\section{Inclusion criteria for collection of studies \\ Type of studies}

The review will include all clinical trials to investigate the central mechanism of acupuncture in the treatment of PDPN based on rs-fMRI using ALFF, ReHo or FC as the main outcomes. The right anterior cingulate cortex, amygdala, hypothalamus and ventrolateral periaqueductal grey will be defined as region of interest for FC. ${ }^{13}$ Other experiments, such as non-clinical trials, noncontrolled trials will not be included.

\section{Type of participants}

This analysis will include studies on adult patients 18 years of age or older who have been diagnosed with PDPN according to the diagnostic criteria from the WHO, the American Diabetes Association and the diagnostic criteria for DPN from the International Association for Pain Research. No constraints will be placed on regional data, educational background, race and gender.

Type of interventions

Patients in the treatment group will be given acupuncture without restrictions on the needle content, choice 
of treatment point, mode of procedure, the retention time of the needle and course of treatment. Interventions without stimulating acupoints combined with two or more therapies or with potential safety problems will be excluded.

\section{Type of outcomes}

\section{Main outcomes}

The main outcomes will include the ALFF, ReHo, FC of the brain, superficial peroneal nerve sensory nerve conduction velocity (SCV), bilateral dorsal current perception threshold (CPT) values of the first toe, the Michigan Diabetic Peripheral Neuropathy Screening Inventory (MNSI), the Neuropathic Pain Symptom Inventory (NPSI), the Leeds Neuropathic Signs and Symptoms Score (LANSS), the Brief Pain Inventory (BPI) and the McGill Pain Questionnaire.

\section{Secondary outcomes}

The secondary outcomes for the study will be fasting glucose, serum C-peptide, blood lipids, glycated serum protein and the depression and anxiety status of the patients with PDPN.

\section{Search strategies for recognising studies \\ Electronic searches}

We will search articles in electronic databases including PubMed, Embase, the Web of Science, Cochrane Library, China National Knowledge Infrastructure, WanFang database, China Science Technology Journal Database (VIP) and the China Doctor/Master Dissertations Fulltext Database. All the publications to 30 June 2021 will be searched. Also, by searching the WHO International Clinical Trials Registry Platform, Chinese Clinical Trial Registry and ClinicalTrials.gov, we will retrieve unpublished protocols and overview findings. Our approach uses a combination of subject and free words and will be decided after several pre-searches. At the same time, the original literature of all the study references will be searched to supplement and obtain relevant literature and ensure a high recovery rate. An example of the search strategy for the Medical Search Headings that will be used is presented in table 1 . For Chinese databases, Chinese translations of these search terms will be used. This approach will adapt and incorporate similar search techniques for other electronic databases.

\section{Other search methods}

Specific conference papers will be manually retrieved and the experts in the field will contact the corresponding authors to obtain valuable information that cannot be accessed by the above data retrieval process.

\section{Data acquisition and analysis}

Selection of studies

Before reviewing the studies, researchers will discuss and decide the screening criteria for the community. The corresponding study members will import the retrieved studies for repetition elimination into the EndNote V.X9 content

\begin{tabular}{|c|c|}
\hline Number & Search terms \\
\hline$\# 1$ & Diabetic neuropathy (MeSH) \\
\hline \#2 & Diabetic neuropathies (MeSH) \\
\hline \#3 & Neuropathies.ti, ab. \\
\hline \#4 & Neuropathy.ti, ab. \\
\hline \#5 & Painful.ti, ab. \\
\hline \#6 & Or\#1-\#5 \\
\hline$\# 7$ & Acupuncture (MeSH) \\
\hline \#8 & Acupuncture therapy (MeSH) \\
\hline$\# 9$ & Acupuncture point.ti, ab. \\
\hline \#10 & Acupuncture ear (MeSH) \\
\hline \#11 & Electroacupuncture.ab. \\
\hline \#12 & Moxibustion.ab. \\
\hline \#13 & Warm acupuncture \\
\hline \#14 & Skin acupuncture \\
\hline \#15 & Acupoints.ti, ab. \\
\hline \#16 & Or \#7-\#15 \\
\hline$\# 17$ & Randomised controlled trial.pt. \\
\hline \#18 & Controlled clinical trial.pt. \\
\hline \#19 & Clinical Trial.pt. \\
\hline \#20 & Trial.ti, ab. \\
\hline \#21 & Clinical trials as topic.sh. \\
\hline \#22 & Pragmatic clinical trial.pt. \\
\hline \#23 & Or \#17-\#22 \\
\hline \#24 & Magnetic Resonance Imaging (MeSH) \\
\hline \#25 & Functional Neuroimaging (MeSH) \\
\hline \#26 & $\begin{array}{l}\text { Functional Magnetic Resonance Imaging } \\
\text { (MeSH) }\end{array}$ \\
\hline \#27 & $\begin{array}{l}\text { Resting state functional magnetic resonance } \\
\text { imaging (MeSH) }\end{array}$ \\
\hline \#28 & Or \#14-\#27 \\
\hline \#29 & \#6 AND \#16 AND \#23AND\#28 \\
\hline \#30 & Remove duplicates from \#29 \\
\hline
\end{tabular}

$\mathrm{MeSH}$, Medical Search Headings.

management system. Ineligible studies will be excluded by reading the titles and abstracts. The final included studies will be determined by reading the full text and discussing the study within the group and contacting the authors for more details if required. Information retrieval and literature screening will be conducted independently by two study members. In cases of disagreement, a third party will be consulted to assess if the study should be included in the final analysis. A description of the selection process is presented in the PRISMA flowchart shown in figure 1.

\section{Data acquisition and management}

Extraction of the research background and design information

Two reviewers will extract the following details based on a pre-designed data extraction form before data 


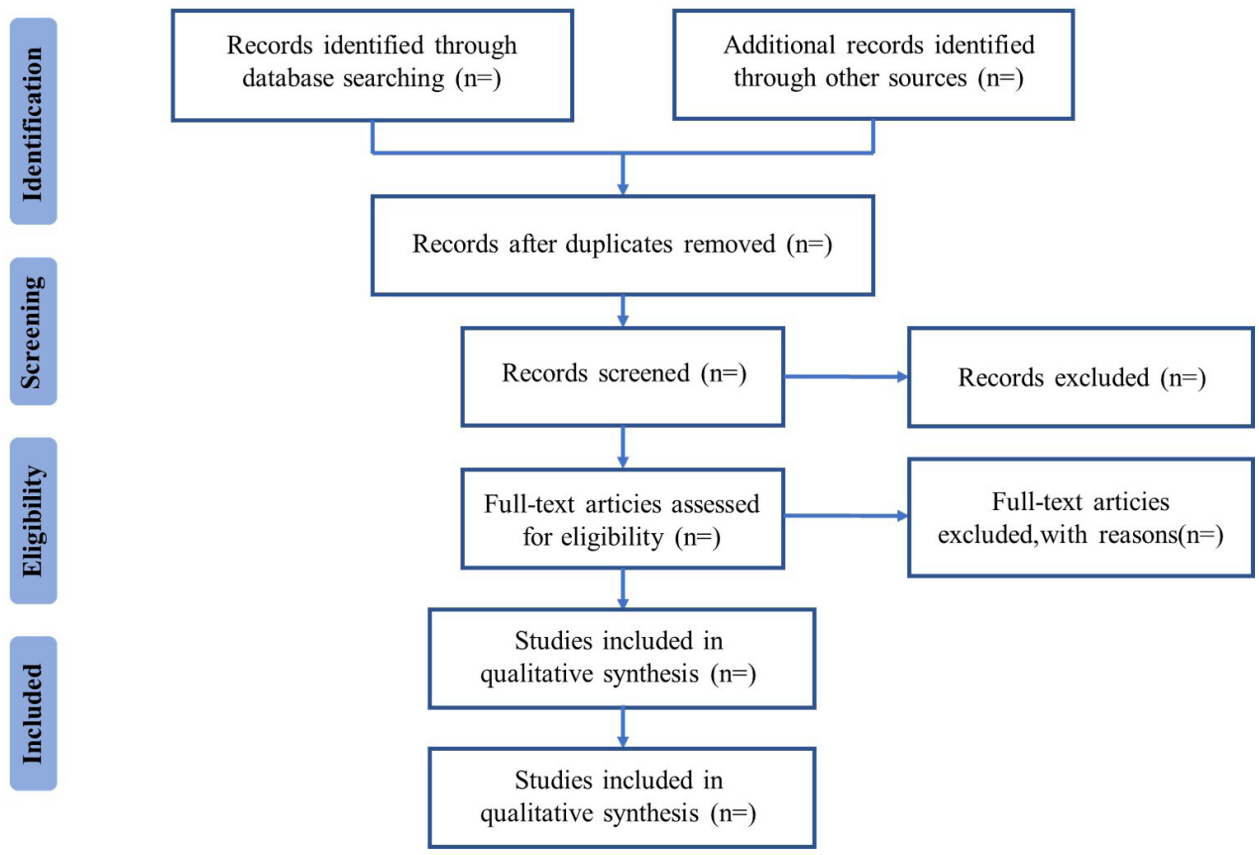

Figure 1 Preferred Reporting Items for Systematic Reviews and Meta-Analyses flow diagram of the study process.

collection: details of the research (name of the first author, year of publication, if it is a multicentre study), details of participants (baseline data, gender, age, PDPN diagnostic criteria, exclusion criteria), methods of study (sample size, distribution hiding, blinding), treatment and control group intervention methods and the primary and secondary outcomes (ALFF, ReHo, FC, SCV, CPT, MNSI, NPSI, LANSS, BPI, fasting glucose, serum C-peptide, blood lipids, glycated serum protein, depression and anxiety status, as well as follow-up duration and adverse events). Any differences will be discussed and consulted through an experienced third party.

\section{Quality control for fMRI data}

The relative head movement of the subjects will be $<2 \mathrm{~mm}$, the time of all functional data included in the study will be $>6 \mathrm{~min}$ and all functional data included in the study will be smoothed. All data preprocessing included in the study must be registered to the Montreal Neurological Institute standard space template. All of the included studies must provide information such as the equipment model, magnetic field strength, voxel size, field of view, repetition time and echo time.

\section{Assessment of risk of bias in included studies}

The risk of bias will be assessed using the Cochrane Risk of Bias Assessment Method, ${ }^{30}$ which focuses on selection (including random sequence generation and allocation concealment), implementation (including blinding of researchers and subjects), evaluation (blinded study outcomes assessment), follow-up (competence of outcome data), reporting (selective study outcomes reporting) and other sources of bias. For a total of seven entries in six fields, the probability of bias will be assessed (other sources of bias). Based on the risk of bias evaluation criteria, 'low risk of bias', 'high risk of bias' and 'unclear' will be calculated for each entry. Review Manager statistical software (RevMan) V.5.4 will produce a graphical presentation of the risk evaluation of bias.

\section{Measures of treatment effects}

For continuous variable results, we will report the mean difference (MD) or standardised MD at the 95\% CI. We will report the relative risk and $95 \%$ CI for dichotomous outcomes.

\section{Dealing with missing data}

In the case of studies where data are missing, the researcher will attempt to obtain information by contacting the corresponding author of the study. We will build our research on the available data if communication is unsuccessful.

\section{Data synthesis and analysis}

The meta-analysis will be performed using RevMan V.5.4 software provided by the Cochrane Collaboration. Before the meta-analysis for effects is performed, we will examine heterogeneity in the data. A standard $\chi^{2}$ test with a significance level of $\mathrm{p}<0.10$ will be used to detect heterogeneity. To measure discrepancies across studies and to evaluate the effect of heterogeneity in meta-analyses, the $\mathrm{I}^{2}$ statistics will be used. ${ }^{31} \mathrm{I}^{2}$ values of $0 \%-30 \%$ indicate low heterogeneity, $30 \%-50 \%$ indicate medium heterogeneity and values $\geq 50 \%$ indicate high heterogeneity. When the data are highly heterogeneous, subgroup analysis will be performed and the reasons for the heterogeneity will be explained. A fixed-effects model will be used for metaanalysis when no statistical heterogeneity is observed between the results. The source of heterogeneity will be further analysed when statistical heterogeneity is detected in the results and after excluding the effects of significant 
clinical heterogeneity, a meta-analysis will be performed using a random-effects model.

\section{Subgroup analysis}

If necessary, the data will be analysed in groups according to different factors such as differences in the acupuncture method, frequency of treatment and the follow-up time.

\section{Sensitivity analysis}

Sensitivity analysis will be used to assess the stability of the meta-analysis results based on the following criteria: study quality, sample size, missing data, quality of heterogeneity, and statistical model.

\section{Ethics and dissemination}

The systematic review and meta-analysis will be based on published literature for data extraction and will not include the use of individual patient data and so no ethical approval is required. The results from this study will be disseminated through conferences and in peerreviewed journals.

\section{DISCUSSION}

DM is a severe metabolic condition characterised by chronic hyperglycaemia and is one of the primary causes of peripheral neuropathy. ${ }^{32}$ Approximately 50\%-60\% of patients with DPN have recurrent pain. ${ }^{33}$ Usually, a stocking-glove pattern that progresses proximally from the feet and hands induces burning pain, paresthesia and numbness. Also, sleep deprivation and depression commonly affect patients with PDPN. ${ }^{34}$ The symptoms associated with PDPN have a major impact on the quality of life of patients. Studies have demonstrated that acupuncture can effectively improve the clinical symptoms of PDPN. In this scenario, acupuncture is a simple and convenient treatment method that has no adverse side effects as so acupuncture therapy is widely used to treat PDPN and improve the quality of life of patients.

Recently, MRI and other brain imaging modalities have demonstrated utility as non-invasive tools to evaluate changes occurring in the brain. The simultaneous development of modern information science and technology in multimodal data fusion and processing analysis methods has shown that brain imaging may play an increasingly important role in revealing the pathogenesis and central mechanisms of PDPN. Rs-fMRI is widely used to study the mechanism of acupuncture in the treatment of neurological diseases. ALFF and ReHo are neuroimaging analysis methods used to measure spontaneous fluctuations in the BOLD-fMRI signal intensity for a given region in the resting brain. ${ }^{35}$ Changes in ALFF and ReHo can be used to locate the spontaneous neural activities in specific regions and the physiological state of the brain. FC can reveal the key roles and regulatory influences on the activity of the target brain area of the entire network. Correlation analysis can be used to calculate the relationship between the average ALFF, ReHo and FC in the changing area of the brain in patients with PDPN and their clinical characteristics. These parameters can reveal the central effect mechanism of acupuncture.

Despite evidence from clinical and animal studies, the central mechanism of acupuncture in the treatment of PDPN has not been elaborated in detail. Furthermore, there has been no systematic evaluation and meta-analysis of this area. In addition to evaluating the improvement of acupuncture treatment for PDPN pain, this study will also explore the latest literature on the mechanism of acupuncture treatment for PDPN. However, there are a relatively small number of studies related to this area of research and our study has some limitations. In summary, this systematic review and meta-analysis aims to elaborate the central nervous mechanism of acupuncture in the treatment of PDPN and will provide a reliable clinical research basis for promoting acupuncture in its treatment.

Contributors The study was designed by HW. ML drafted the manuscript and the protocol which was revised by LY. All authors developed the search strategy and that will be run by $\mathrm{HH}, \mathrm{HZ}$ and $\mathrm{JC}$. $\mathrm{HH}, \mathrm{HZ}$ and $\mathrm{JC}$ will also independently screen the prospective studies, extract data from included studies and determine the possibility of bias. The data synthesis will be performed by GW and YL. In cases of disputes, ML and LY will arbitrate and ensure that no errors occur during the analysis. The publication of the protocol has been authorised by all authors.

Funding This work was supported by the National Natural Science Foundation of China, grant number 81874502 and 82074548 .

Competing interests None declared.

Patient and public involvement Patients and/or the public were not involved in the design, or conduct, or reporting, or dissemination plans of this research.

Patient consent for publication Not required.

Provenance and peer review Not commissioned; externally peer reviewed.

Open access This is an open access article distributed in accordance with the Creative Commons Attribution Non Commercial (CC BY-NC 4.0) license, which permits others to distribute, remix, adapt, build upon this work non-commercially, and license their derivative works on different terms, provided the original work is properly cited, appropriate credit is given, any changes made indicated, and the use is non-commercial. See: http://creativecommons.org/licenses/by-nc/4.0/.

ORCID iDs

Mengyuan Li http://orcid.org/0000-0002-0039-6661

Lin Yao http://orcid.org/0000-0002-5948-2249

\section{REFERENCES}

1 Pop-Busui R, Boulton AJM, Feldman EL, Rodica P-B, Boulton Andrew JM, et al. Diabetic neuropathy: a position statement by the American diabetes association. Diabetes Care 2017;40:136-54.

2 Malik RA. Why are there no good treatments for diabetic neuropathy? Lancet Diabetes Endocrinol 2014;2:607-9.

3 Shahram B, Kourosh Z, Bibi SH. Comparison of the accuracy of monofilament testing at various points of feet in peripheral diabetic neuropathy screening. J Diabetes Metab Disord 2014;13:1-7.

4 Abbott CA, Malik RA, van Ross ERE, et al. Prevalence and characteristics of painful diabetic neuropathy in a large communitybased diabetic population in the U.K. Diabetes Care 2011;34:2220-4.

$5 \mathrm{Kim}$ JM, Son CG, Cho CS. Review of randomized controlled clinical trials targeting treatment of diabetic peripheral neuropathy. $J$ Korean Oriental Med 2010;31:164-70.

6 Bril V, England J, Franklin GM. American Academy of Neurology; American association of neuromuscular and Electrodiagnostic medicine; American Academy of physical medicine and rehabilitation. evidence-based guideline: treatment of painful diabetic neuropathy. Neurology 2011;76:1758-65. 
7 Dobrota VD, Hrabac P, Skegro D. The impact of neuropathic pain and other comorbidities on the quality of life in patients with diabetes. . Health \& Quality of Life Outcomes, 2014: 12. 1-8.

8 Tesfaye S, Selvarajah D, Gandhi R. Diabetic peripheral neuropathy may not be as its name suggests: evidence from magnetic resonance imaging[J]. Pain 2016;157Suppl 1:S72-80.

9 Tadic M, Cuspidi C, Sala C. COVID-19 and diabetes: is there enough evidence? J Clin Hypertens 2020;22:943-8.

10 L S R, Chang KH. Neuropathic pain: mechanisms and treatments. Biomed J 2005;28:597-605.

11 Veves A, Backonja M, Malik RA. Painful diabetic neuropathy: epidemiology, natural history, early diagnosis, and treatment options. Pain Med 2008;9:660-74.

12 Boadas-Vaello P, Homs J, Reina F, et al. Neuroplasticity of supraspinal structures associated with pathological pain. Anat Rec 2017;300:1481-501.

13 Segerdahl AR, Themistocleous AC, Fido D, et al. A brain-based pain facilitation mechanism contributes to painful diabetic polyneuropathy. Brain 2018;141:357-64.

14 Argoff CE, Backonja M-M, Belgrade MJ, et al. Consensus guidelines: treatment planning and options. Mayo Clinic Proceedings 2006;81:S12-25

15 Gillespie EA, Gillespie BW, Stevens MJ. Painful diabetic neuropathy: impact of an alternative approach. Diabetes Care 2007;30:999-1001.

16 Abuaisha BB, Costanzi JB, Boulton AJ. Acupuncture for the treatment of chronic painful peripheral diabetic neuropathy: a longterm study. Diabetes Res Clin Pract 1998;39:115-21.

17 Dimitrova A, Murchison C, Oken B. Acupuncture for the treatment of peripheral neuropathy: a systematic review and meta-analysis. $J$ Altern Complement Med 2017;23:164-79.

18 Ding SS, Hong SH, Wang C, et al. Acupuncture modulates the neuroendocrine-immune network. QJM 2014;107:341-5.

19 Bailey A, Wingard D, Allison M, et al. Acupuncture treatment of diabetic peripheral neuropathy in an American Indian community. $J$ Acupunct Meridian Stud 2017;10:90-5.

20 Kasuya D. [Acupuncture for painful diabetic neuropathy]. Rinsho Shinkeigaku 2012;52:1290-3.

21 Zang Y-F, He Y, Zhu C-Z, et al. Altered baseline brain activity in children with ADHD revealed by resting-state functional MRI. Brain Dev 2007;29:83-91.
22 Biswal B, Yetkin FZ, Haughton VM, et al. Functional connectivity in the motor cortex of resting human brain using echo-planar MRI. Magn Reson Med 1995;34:537-41.

23 Zang Y, Jiang T, Lu Y, et al. Regional homogeneity approach to fMRI data analysis. Neuroimage 2004;22:394-400.

24 Wu J-J, Lu Y-C, Hua X-Y, et al. Cortical remodeling after electroacupuncture therapy in peripheral nerve repairing model. Brain Res 2018;1690:61-73.

25 Wang Z, Yan C, Zhao C, et al. Spatial patterns of intrinsic brain activity in mild cognitive impairment and Alzheimer's disease: a resting-state functional MRI study. Hum Brain Mapp 2011;32:1720-40.

26 Fan Z, Chen X, Qi Z-X, et al. Physiological significance of R-fMRI indices: can functional metrics differentiate structural lesions (brain tumors)? Neuroimage Clin 2019;22:101741.

27 Qiu L, Tan X, Zou M, et al. [Changes in regional homogeneity of brain activity in patients with diabetic peripheral]. Nan Fang Yi Ke Da Xue Xue Bao 2018;38:1433-9.

28 Yang S-Q, Xu Z-P, Xiong Y, et al. Altered Intranetwork and Internetwork functional connectivity in type 2 diabetes mellitus with and without cognitive impairment. Sci Rep 2016;6:1-11.

29 Shamseer L, Moher D, Clarke M, et al. Preferred reporting items for systematic review and meta-analysis protocols (PRISMA-P) 2015: elaboration and explanation. BMJ 2015;349:g7647.

30 Higgins JPT, Altman DG, Gotzsche PC, et al. The Cochrane Collaboration's tool for assessing risk of bias in randomised trials. BMJ 2011;343:d5928.

31 Higgins JPTet al. Measuring inconsistency in meta-analyses. BMJ 2003; $327: 557-60$.

32 Gylfadottir SS, Weeracharoenkul D, Andersen ST, et al. Painful and non-painful diabetic polyneuropathy: clinical characteristics and diagnostic issues. J Diabetes Investig 2019;10:1148-57.

33 Bair MJ, Brizendine EJ, Ackermann RT, et al. Prevalence of pain and association with quality of life, depression and glycaemic control in patients with diabetes. Diabet Med 2010;27:578-84.

34 Ziegler D, Ametov A, Barinov A, et al. Oral treatment with alpha-lipoic acid improves symptomatic diabetic polyneuropathy: the Sydney 2 trial. Diabetes Care 2006;29:2365-70.

35 Cordes D, Haughton VM, Arfanakis K, et al. Frequencies contributing to functional connectivity in the cerebral cortex in "resting-state" data. AJNR Am J Neuroradiol 2001;22:1326-33. 